\title{
A FOURTEEN YEARS OLD BOY WITH CHOLESTEROL ESTER STORAGE DISEASE
}

\author{
FAIZUL ISLUM CHOWDHURY ${ }^{1}$, AHMEDUL KABIR ${ }^{2}$, JAYANTA BANIK ${ }^{3}$, PINAKI PAUL ${ }^{4}$, MOSTOFA \\ KAMAL $^{5}$, HASNA HENA PARVEEN ${ }^{6}$, UMME KULSUM MITU ${ }^{7}$, ROWZATUL FERDOUS ${ }^{8}$
}

\begin{abstract}
Cholesterol ester storage disease (CESD) is a rare autosomal recessive disorder resulting from lysosomal acid lipase deficiency and is usually characterized by hepatomegaly and hyperlipidemia. It is diagnosed by liver biopsy which characteristically shows microvesicular steatosis and periportal fibrosis. Here we report a fourteen years old boy who had presented with unexplained hepatomegaly, and hyperlipidemia determined incidentally. He was finally diagnosed as a case of cholesterol ester storage disease by liver biopsy. Though there is yet no specific treatment for CESD; however, the early detection of cases would make the timely control of complications possible.
\end{abstract}

Keyword: Cholesterol Ester Storage Disease, Lysosomal Storage Diseases, Lipidoses

\section{Introduction}

Cholesterol ester storage disease (CESD), is a rare autosomal recessive lysosomal storage disorder which is infrequent in children. ${ }^{1}$ It is due to the deficiency of lysosomal acid lipase (LAL), which is present in lysosomes of all nucleated cells and catalyzes the hydrolysis of highly hydrophobic cholesterol esters and triglycerides. ${ }^{2}$ Acid lipase deficiency in the leukocytes were demonstrated in $50 \%$ of parents of the affected children. ${ }^{1}$ There is massive accumulation of cholesterol esters and triglycerides in many organs, particularly the liver as a result of deficient activity of LAL. ${ }^{2}$ The accumulated cholesterol esters are mainly derived from serum LDL. ${ }^{3}$ There are two forms of LAL deficiency; one is Wolman disease which is the severe clinical form and is a fatal disorder of infancy, whereas the another form, CESD is a milder late-onset form, usually characterized by hepatomegaly and hypercholesterolemia. ${ }^{2}$

There is no specific routine laboratory observations to diagnose the disease. The mainstay of diagnosis is based on the the combination of hepatomegaly with accumulation of macrophages and ultrastructural evidence of lysosomal lipid storage. ${ }^{4,5}$ There is a high probability of a underdiagnosis as nonalcoholic steatohepatitis (NASH) as it has a higher incidence than CESD. ${ }^{4}$

\section{Case report}

A 14 years old boy presented to our institution with fever and pain in the right lower chest and right upper arm for six days. He also noticed the presence of a lump in the right upper abdomen since his six years of age. Review of symptoms revealed a history of frequent syncopal attack during micturition and defecation. There is also history of generalized weakness and respiratory distress during playing since childhood. There is no significant past medical history and family history is unremarkable.

On examination, he was found to be febrile with Temperature: $39^{\circ} \mathrm{C}$. He was hemodynamically stable with mild anaemia and no lymphadenopathy. Examination of the chest reveals dull percussion note and diminished breath sound in the right lower chest. Abdominal examination reveals hepatomegaly of about

1. Associate Professor, Dept. of Medicine, Dhaka Medical College, Dhaka

2. Assistant Professor, Dept of Medicine, Dhaka Medical College, Dhaka

3. Post-graduate Trainee, Dept. of Medicine, Bangabandhu Sheikh Mujib Medical University, Dhaka

4. Registrar, Dept. of Medicine, Dhaka Medical College Hospital, Dhaka

5. Assistant Registrar, Dept. of Medicine, Dhaka Medical College Hospital, Dhaka

6. Medical Officer, Dept. of Medicine, Dhaka Medical College Hospital, Dhaka

7. Post-graduate Trainee, Dept. of Medicine, Dhaka Medical College Hospital, Dhaka

8. Intern Doctor, Dept. of Medicine, Dhaka Medical College Hospital, Dhaka

Correspondence : Dr. Ahmedul Kabir, Assistant Professor, Dept of Medicine, Dhaka Medical College, Dhaka 
$8 \mathrm{~cm}$ from the right costal margin which is nontender, has a sharp margin, smooth surface and firm consistency. There is no other organomegaly.

On investigation, Complete blood count and Blood film reveals, $\mathrm{WBC}-15 \mathrm{X} 10^{9} / \mathrm{L}$ with a PMN predominance of $75 \%$, Hematocrit $-43 \%$, Platelets $-286 \times 10 x^{9} / \mathrm{L}$, ESR $-70 \mathrm{~mm} / 1^{\text {st }} \mathrm{hr}, \mathrm{Hb}$ ( Cyn. Meth. ) $-9.6 \mathrm{~g} / \mathrm{dl}$, PBF : RBC - Microcytic and hypochromic, WBC Mature with Neutrophil Leukocytosis, Plateletes Normal. Urinalysis is negative for ketone bodies, protein or red cells. LFT shows, S.Bilirubin $-0.8 \mathrm{mg} /$ dl, ALT - 20 U/L, AST - 18 U/L, Prothrombin time $12.5 \mathrm{sec}$ ( control $12 \mathrm{sec}$ ), S. Total protein $-8.8 \mathrm{gm} / \mathrm{dl}$, S. Albumin $-3.8 \mathrm{gm} / \mathrm{dl}$, S. Globulin $-5.0 \mathrm{gm} / \mathrm{dl}$ and $\mathrm{S}$. AG ratio $-0.8: 1$. Fasting blood glucose is 3.56 $\mathrm{mmol} / \mathrm{L}$. Fasting lipid profile shows S. Total Cholesterol $-224.0 \mathrm{mg} / \mathrm{dl}$, S. HDL $-28.0 \mathrm{mg} / \mathrm{dl}$, S. $\mathrm{LDL}-170.41 \mathrm{mg} / \mathrm{dl}$ and $\mathrm{S}$. TG $-366.0 \mathrm{mg} / \mathrm{dl}$. USG of whole abdomen shows hepatomegaly with homogenous paranchymal echo and mild right sided pleural effusion. Chest radiograph shows small right sided pleural effusion. ECG and Echo is within normal limit. Endoscopy of upper GIT is normal. Liver biopsy shows that the lobular architecture is lost and is replaced by nodule formation containing hepatocytes. The hepatocytes are large showing ballooning degenation while others show moderate steatosis. The nodules are separated by thick fibrous septae. Portal areas are expanded and reveal moderate infiltration of chronic inflammatory cells with mild fibrosis. Mild piecemeal necrosis is also seen. PAS stain and PAS-D is negative.

\section{Discussion}

Abnormal accumulation of cholesterol esters and triglycerides resulting from lysosomal acid lipase deficiency, occurs in many tissues. This is expressed in two forms, namely, Wolman disease (WD) and cholesterol ester storage disease. Both are autosomal recessive disorders. Among them the WD is fatal and manifests before the age of one year. The CESD is a milder form that usually manifests in adulthood. ${ }^{6}$

The biochemical defect is in the lysosomal acid cholesterol hydrolase (lipase) enzyme, the gene of which is located on chromosome 10q23.2-q23.3. It contains 10 exons dispersed over 45 kilobases. Several mutations have been identified, some of which lead to almost complete loss of enzyme activity and to the phenotype of Wolman's disease, while other mutations cause only partial disruption of the enzyme action, leading to a clinically more benign disease, the cholesterol ester storage disease. ${ }^{6}$ In this situation, the LDL, triglycerides and cholesterol esters that enter the cells cannot be hydrolyzed, due to the deficiency of lysosomal acid lipase resulting in the massive accumulation of cholesterol esters and triglycerides especially in the liver, but also in the spleen, adrenal glands, lymph nodes, intestinal mucosa, vascular endothelium, and muscle. ${ }^{2,7}$

CESD is usually presented with hepatomegaly, hyperlipidemia or elevated transaminase levels and is found coincidentally during examination for other health problems. ${ }^{2}$ Other clinical features of CESD include splenomegaly, adrenal calcifications, premature atherosclerosis, diarrhea, steatorrhea, failure to thrive, and neurologic disturbances. ${ }^{2}$ Our case had only hepatomegaly, determined incidentally as patient comes with fever and pain in the right lower chest and right upper arm for a short duration; the rest of the physical examination was normal except right sided mild pleural effusion.

Increased transaminases, high cholesterol and lowdensity lipoprotein (LDL), reduced high-density lipoprotein (HDL), and variable triglyceride levels are found in most of the patients with CESD. Hepatosplenomegaly, increased echogenicity of the hepatic parenchyma, and rarely adrenal calcification can be found in abdominal ultrasonography . ${ }^{2}$ Our case had hypercholesterolemia and hypertriglyceridemia with increased LDL cholesterol levels. Also, ultrasonographic examination showed hepatomegaly.

Glycogen storage disease may be easily confused with CESD., Marked hepatomegaly and hyperlipidemia appear in both disorders, but laboratory tests (i.e. hypoglycemia and lactic acidosis in glycogen storage disease), liver biopsy and enzymatic analyses can differentiate them. ${ }^{2}$

Nonalcoholic fatty liver disease (NAFLD) which is also called Nonalcoholic Staetohepatitis (NASH) can also be confused with CESD as it the most common spectrum of metabolic liver disease in the world. It encompasses simple steatosis to steatohepatitis, advanced fibrosis, cirrhosis and hepatocellular carcinoma. Liver biopsy remains the most specific and sensitive method to differentiate NAFLD, which provides important information on the long-term prognosis of the patients. ${ }^{9}$ 
It is very difficult to differentiate CESD and Wolman disease through microscopic examination of liver biopsy even with electron microscopic examination. Enlarged and vacuolated hepatocytes and Kupffer cells, periportal fibrosis and cirrhosis may be seen in liver histology in both cases. But the extensive bilateral punctate calcification throughout the adrenals, with retention of their normal triangular shape is diagnostic of WD. ${ }^{6}$ In Wolman disease there is also infiltration of fat cells in the bone marrow, lymphnode and intestinal mucosa. ${ }^{6}$

Vacuolation of hepatocytes as seen by light microscopy can also be present in Niemann- Pick disease type B, mucopolysaccharidoses, and GM1 gangliosidosis . Clinical findings and enzyme assays can help to distinguish them from CESD. ${ }^{2}$ In CESD, histological examination of liver biopsy specimens shows heatsensitive birefringent crystals of cholesterol in liver tissue in their frozen sections which is not observed in other lysosomal storage disorders. ${ }^{2,8}$

The diagnosis of CESD is based on clinical picture (hepatomegaly), biochemical abnormalities (elevated serum aminotransferase activities and hyperlipoproteinemia) and liver biopsy findings (accumulation of fat within hepatocytes, bile duct epithelium endothelial and Kupffer cells with birefringence stain of cholesterol crystals in frozen sections). Demonstration of lysosomal acid lipase deficiency in liver, cultured skin fibroblasts or peripheral mononuclear leukocytes confirms the diagnosis. ${ }^{2,4,8}$ Our patient fulfill all the criteria along with histologic featuers of cholesterol ester storage disease, but we cannot assess the lysosomal acid lipase activity due to technical limitations.

There is no specific treatment for cholesterol ester storage disease. The main treatment options are supportive therapies with low-cholesterol diet, fat soluble vitamin supplements, and cholestyramine. 3hydroxy 3-methyl glutaryl (HMG) CoA reductase inhibitors are used for hyperlipoproteinemia. ${ }^{2}$ Simvastatin and Lovastatin were used in different cases for short time. Though they were well tolerated without side effects, but no significant changes were seen in serum lipoprotein concentrations or liver histopathology after short term therapy. ${ }^{2,8}$ Therefore long-term follow-up is needed to observe the possible side effects due to medication and also progression of the disease. ${ }^{2}$ For progressive liver disease liver transplantation is the choice of treatment. In future enzyme replacement and gene therapy might be possible. $^{2}$

\section{Conclusion}

Though cholesterol ester storage disease is a rare disorder it should be suspected in cases of unexplained hepatomegely. There is no specific test to diagnose the case and the diagnosis is based on clinical picture, biochemical abnormalities and liver biopsy findings. The main goal of treatment is the prevention of possible complications.

\section{Reference}

1. D'Agostino D, Bay L, Gallo G, Chamoles N. Cholesterol ester storage disease: clinical, biochemical, and pathological studies of four new cases. J Pediatr Gastroenterol Nutr 1988;7(3):44650.

2. Dalgýç B, Sar S, Gündüz M, Ezgü F, et al. Cholesteryl ester storage disease in a young child presenting as isolated hepatomegaly treated with simvastatin. The Turkish Journal of Pediatrics 2006; 48(2):148-151.

3. Todoroki T, Matsumoto K, Watanabe K, et al. Accumulated lipids, aberrant fatty acid composition and defective cholesterol ester hydrolase activity in cholesterol ester storage disease. Annals of Clinical Biochemistry 2000;37(2):187-193.

4. Weiler, Freudenberg F, Müller-Höcker J. Cholesterol ester storage disease-A rare disease or a rare diagnosis? 2009;30(1):65-69.

5. Boldrini R, Devito R, Biselli R, et al. Wolman disease and cholesteryl ester storage disease diagnosed by histological and ultrastructural examination of intestinal and liver biopsy. Pathology - Research and Practice 2004; 200(3):231240.

6. Essa MA, Nounou R, Sakati N, et al. Wolman's disease: the king faisal specialist hospital and research centre experience. Ann Saudi Med 1998; 18(2):120-124.

7. Yousef TM, Shafik MH, Shalan YAF. Wolman Disease in an Egyptian Patient. Kuwait Medical Journal 2005;37(3): 200-202.

8. Di Bisceglie AM, Ishak KG, Rabin L, Hoeg JM. Cholesteryl ester storage disease: hepatopathology and effects of therapy with lovastatin. Hepatology. 1990;11(5):764-72.

9. Sevastianos VA, Hadziyannis SJ. Nonalcoholic fatty liver disease: from clinical recognition to treatment. Expert Review of Gastroenterology \& Hepatology 2008;2(1):59-79. 